\title{
Breastfeeding Intentions and Practice among Vegetarians in the United States - A Pilot Study
}

\author{
Armstrong AM and Anderson $\mathrm{AK}^{*}$ \\ Department of Foods and Nutrition, University of Georgia, Athens, USA
}

${ }^{*}$ Corresponding author: Anderson $\mathrm{AK}, \mathrm{PhD}, \mathrm{MPH}, \mathrm{CPH}$, Department of Foods and Nutrition, University of Georgia, 280 Dawson Hall, Athens, GA, USA 30602, Tel: (706) 542-5059, E-mail: fianko@uga.edu

Citation: Armstrong AM, Anderson AK (2015) Breastfeeding Intentions and Practice among Vegetarians in the United States - A Pilot Study. J Nutr Health Sci 2(3): 301. doi: 10.15744/2393-9060.2.301

Received Date: May 09, 2015 Accepted Date: August 25, 2015 Published Date: August 28, 2015

\begin{abstract}
Background: Vegetarianism is a rapidly growing practice in the United States that is remarkably understudied. Currently, no study has assessed the infant feeding behaviors among vegetarians or the sub-types to determine their breastfeeding patterns.

Objectives: This study assessed breastfeeding intentions and previous breastfeeding experiences of vegetarians as well as the differences in breastfeeding intentions and previous breastfeeding experiences between the different vegetarian groups in the United States.

Methods: This was a cross-sectional online survey of female vegetarians. The survey link was emailed to administrators of vegetarian listservs for their membership to complete. The outcome measures assessed included breastfeeding intentions and actual breastfeeding practices. Descriptive statistics were used to summarize the outcome variables and other variables of interest, and results reported as percentages and mean \pm SD. Binary logistic regression was used to identify predictors of breastfeeding intentions and practice. P-value of $<0.05$ was used as criterion for statistical significance.

Results: Of the 265 vegetarians completing the online survey, $38.5 \%$ were vegans, $30.6 \%$ lacto-ovo vegetarians, $10.9 \%$ pesco-vegetarians, 8.3\% flexitarians, $7.2 \%$ lacto-vegetarians, and $4.5 \%$ ovo-vegetarians. Overall, participants' breastfeeding intentions, including exclusive breastfeeding, were higher than actual breastfeeding practice, irrespective of vegetarian type. There was no significant difference between breastfeeding intentions and practices by vegetarian type.

Conclusion: The majority of vegetarians who participated in this study and the various sub-types intended to breastfeed, and did breastfeed. Future studies of more heterogeneous and larger sample-size are needed to accurately ascertain the breastfeeding practices of the various vegetarian groups.

Keywords: Vegetarian; Breastfeeding; Online survey; Pesce; Vegan; Flexitarian
\end{abstract}

\section{Background}

Breastfeeding is indisputably the preferred method of optimum infant feeding for the first six months of life [1,2]. National and international organizations including the Academy of Nutrition and Dietetics[2]; the American Academy of Pediatrics [1]; the World Health Organization [3,4]; and the blueprint for the health of Americans, Healthy People 2020; support breastfeeding and strive to increase the breastfeeding rate among mothers in the United States $[5,6]$.

The term vegetarian refers to a person whose diet primarily consists of grains, legumes, nuts, seeds, vegetables, and fruits [7]. Pesce vegetarians include fish in the basic vegetarian diet, whereas flexitarians consume a normal vegetarian diet, but will occasionally consume organic meat and other animal products [7]. Lacto vegetarians include dairy products in their basic vegetarian diet [7]. Ovo vegetarians, consume eggs in addition to the basic vegetarian diet. Lacto-ovo vegetarians include both dairy products and eggs to the basic vegetarian diet. Vegans maintain the basic vegetarian staple diet, but exclude all animal products and animal derived substances: eggs, dairy products, and even in some cases, honey [7].

Whereas women who practice vegetarianism constitute a minority group in the United States, they tend to be well educated, of higher-income status, and of a healthy BMI [8-10], characteristics similar to the general breastfeeding mothers in the United States. Surprisingly, no study to date has examined the relationship between vegetarianism and breastfeeding. With the increasing prevalence of vegetarianism in the United States, about 1\% in 1971 to $13 \%$ in 2013 [11-14], and efforts by health organizations to improve breastfeeding rates, understanding the influence that vegetarianism has on breastfeeding intentions and practices vegetarian women is important. Currently, there are no studies that have focused on the breastfeeding behaviors of vegetarian mothers. The purpose of this study is to fill an important gap in the literature regarding infant feeding intentions and practices among vegetarians in the United States. 


\section{Methods}

\section{Study Design and Subjects}

This was a web-based cross-sectional survey of a convenience sample of vegetarian women in the United States.

A total of 293 female self-identified vegetarians responded to the online survey; however, 28 of them were excluded because of incomplete data, leaving a total of 265 participants who completed the survey in the final analysis. The study protocol was approved by the Institutional Review Board for Human Subjects at the University of Georgia, and all participants digitally consented to study participation.

Questionnaire: The questionnaire, consisting of 25 questions, was hosted and distributed via the Qualtrics Software. The survey gathered information about dietary practices, demographics, smoking and alcohol use, infant feeding intentions and practices and residential location within the United States. The participants self-identified themselves by choosing from a list of the different types of vegetarianism namely, vegan, fruitarian, lacto-vegetarian, lacto-ovo-vegetarian, ovo-vegetarian, pesce-vegetarian, pollovegetarian, flexitarian and other (write in) that best describes them. Breastfeeding intention was measured via the question "if you are pregnant, or plan on becoming pregnant, what is your primary infant feeding intentions for the first six months after delivery" and the options to choose from included the following four (4) responses: breastfeeding only; formula feeding only; mixed feeding (breast milk and formula); I am not sure, or not decided. Actual breastfeeding practice was assessed with the question "if you have been pregnant and delivered before how did you feed your child" and the options to choose from included the following responses: exclusively breastfeed for up to 3 months; exclusively breastfeed for up to 6 months; mixed feeding for up to 3 months; mixed feeding for up to 6 months; mixed feeding for up to 9 months; mixed feeding for up to 12 months; I did not breastfeed my infant (only formula feeding).

Survey Distribution: On January 30, 2013, survey invitations using the Qualtrics Software were emailed to administrators of vegetarian listservs across the United States (identified through online sources such as websites and magazines) to share with their membership, as well as individually identified vegetarians by word of mouth. After the initial email invitation, 2 follow-up email reminders were sent to the listserv administrators to encourage their members to complete the survey. The survey closed on August 30, 2013.

Before beginning the survey, each participant had the opportunity to read the consent form and agree to participation by checking a box to electronically consent to participation. Once the participant clicked "Yes" to "I agree to participate in this survey" after reading the description for the survey, the survey opened for them to complete.

Statistical Analyses: Data from the online survey was directly imported and analyzed using Statistical Package for the Social Sciences (SPSS) for Windows version 21.0. Descriptive statistics were used to summarize the outcome and other variables of interest. We used chi-square test $\left(\chi^{2}\right)$ to examine the crude relationships between breastfeeding intentions, practice and type of vegetarianism. Due to the small sample of participants, responses were re-coded to combine "exclusive breastfeeding" (defined as feeding breast milk only) and "mixed feeding" (combination of breast milk and formula) as breastfeeding, because no differences were observed when coded separately. Breastfeeding was then compared to formula feeding. Binary logistic regression analysis was used to explore the crude likelihood of intention to breastfeed and previous breastfeeding experience between the vegans and the other vegetarian types together due to the small numbers in each of the subtypes. Results are reported as odds ratios (OR) and the respective $95 \%$ confidence interval (CI). Criterion for statistical significance was set at $\mathrm{p}<0.05$.

\section{Results}

\section{Characteristics of Participants}

The average age of the participants was $33.8 \pm 11.1$ years. Most $(81.1 \%)$ of the participants had at least bachelor's degree with mean years of formal education being $16.2 \pm 4.8$ years, while $63.8 \%$ were either married or cohabiting. The majority (77.0\%) of the participants were Caucasian, 1.7\% Asian/Pacific Islander, 5.3\% Hispanic/Latino while only 0.8\% self-identified as Black/African American and 5.3\% other (including those of mixed race/ethnicity). Eighty percent (80\%) worked full- or part-time. While only $3.8 \%$ of the participants smoked, $58.1 \%$ consumed alcohol. Most $(38.5 \%)$ of the participants identified themselves as vegan, $30.6 \%$ lacto-ovo vegetarian, $10.9 \%$ pesce-vegetarian, $8.3 \%$ flexitarian, $7.2 \%$ lacto-vegetarian and $4.5 \%$ ovo-vegetarian. Participant responses were received from 38 of the 50 States and the District of Columbia in the United States. Reasons for vegetarianism included $45.7 \%$ ethical, $19.6 \%$ health, $15.5 \%$ environmental, $10.9 \%$ aesthetics and $8.3 \%$ religious.

Of the 265 participants, 69 had no plans of giving birth in the future either due to their age or personal choice. Almost threefourths $(72.5 \%)$ of the participants were breastfed as children. Of the 196 who plan to give birth in the future, $78.1 \%$ intend to breastfeed, $1.5 \%$ intend to formula feed, while $20.4 \%$ were undecided on their infant feeding intentions. For participants who reported previous infant feeding experience, $89.0 \%$ initiated breastfeeding of which $59.0 \%$ exclusively breastfed for 6 months, while $11.0 \%$ exclusively formula fed. 


\section{Infant Feeding Intentions and Practices}

Table 1 shows that breastfeeding intentions among vegetarians in this study are relatively high except for flexitarians. Interestingly, two-fifth of the flexitarians was undecided about their infant feeding intentions compared to just about $20 \%$ or less of the other vegetarian groups. Over half (53.8\%) of participants with an associate degree were undecided about their infant feeding intentions, majority of those with bachelor's or higher degrees intend to breastfeed should they give birth. Overall, breastfeeding intention was high among participants in this study (Table 1). Of the vegans who intend to breastfeed, $79.1 \%$ intend to exclusively breastfeed (EBF) for 6 months, while 4.5\% intend to mix feed for 6 months with the rest undecided. About $83.3 \%$ of pesce-vegetarians intend to EBF for 6 months with only $8.3 \%$ undecided about their infant feeding intentions.

\begin{tabular}{|c|c|c|c|c|c|}
\hline & \multicolumn{3}{|c|}{ Infant Feeding Intentions $(n=196)$} & \multicolumn{2}{|c|}{$\begin{array}{c}\text { Previous Breastfeeding Experience (n } \\
=100)\end{array}$} \\
\hline & $\begin{array}{l}\text { Breastfeeding } \\
\text { n }(\%)\end{array}$ & $\begin{array}{l}\text { Formula Feeding } \\
\text { n (\%) }\end{array}$ & $\begin{array}{l}\text { Undecided } \\
\text { n (\%) }\end{array}$ & $\begin{array}{l}\text { Breastfeeding } \\
\text { n }(\%)\end{array}$ & $\begin{array}{l}\text { Formula Feeding } \\
\text { n }(\%)\end{array}$ \\
\hline $\begin{array}{c}\text { Type of Vegetarian } \\
\text { Vegan } \\
\text { Lacto-vegetarian } \\
\text { Ovo-vegetarian } \\
\text { Lacto-ovo vegetarian } \\
\text { Pesco-vegetarian } \\
\text { Flexitarian }\end{array}$ & $\begin{array}{l}55(83.3) \\
14(87.5) \\
7(77.8) \\
47(73.4) \\
20(83.3) \\
10(58.8)\end{array}$ & $\begin{array}{l}0(0.0) \\
1(6.3) \\
0(0.0) \\
2(3.1) \\
0(0.0) \\
0(0.0)\end{array}$ & $\begin{array}{c}11(16.7) \\
1(6.3) \\
2(22.2) \\
15(23.4) \\
4(16.7) \\
7(41.2)\end{array}$ & $\begin{array}{l}38(86.4) \\
4(80.0) \\
4(80.0) \\
32(94.1) \\
5(83.3) \\
6(100.0)\end{array}$ & $\begin{array}{l}6(13.6) \\
1(20.0) \\
1(20.0) \\
2(5.9) \\
1(16.7) \\
0(0.0)\end{array}$ \\
\hline $\begin{array}{l}\text { Educational Level } \\
\leq \text { High school } \\
\text { Associate degree } \\
\text { Bachelor's degree } \\
\text { Master's degree } \\
\text { Doctoral degree }\end{array}$ & $\begin{array}{l}16(76.2) \\
6(46.2) \\
57(82.6) \\
48(80.0) \\
26(78.8)\end{array}$ & $\begin{array}{l}0(0.0) \\
0(0.0) \\
1(1.4) \\
2(3.3) \\
0(0.0)\end{array}$ & $\begin{array}{c}5(23.8) \\
7(53.8) \\
11(15.9) \\
10(16.7) \\
7(21.2)\end{array}$ & $\begin{array}{c}13(86.7) \\
8(88.9) \\
24(85.7) \\
34(89.5) \\
10(100.0)\end{array}$ & $\begin{array}{l}2(13.3) \\
1(11.1) \\
4(14.3) \\
4(10.5) \\
0(0.0)\end{array}$ \\
\hline $\begin{array}{c}\text { Race/Ethnicity } \\
\text { Caucasian } \\
\text { Hispanic/Latino } \\
\text { Asian/Pacific Islander } \\
\text { Black/African American } \\
\text { Other }\end{array}$ & $\begin{array}{c}112(79.4) \\
8(61.5) \\
22(78.6) \\
2(100.0) \\
9(75.0)\end{array}$ & $\begin{array}{c}3(2.1) \\
0(0.0) \\
0(0.0) \\
0(0.0 \\
0(0.0)\end{array}$ & $\begin{array}{c}26(18.4) \\
5(38.5) \\
6(21.4) \\
0(0.0) \\
3(2.5)\end{array}$ & $\begin{array}{c}74(88.1) \\
1(50.0) \\
10(100.0) \\
1(100.0) \\
3(100.0)\end{array}$ & $\begin{array}{c}10(11.9) \\
1(50.0) \\
0(0.0) \\
0(0.0) \\
0(0.0)\end{array}$ \\
\hline $\begin{array}{c}\text { Marital Status } \\
\text { Single } \\
\text { Married } \\
\text { Cohabiting }\end{array}$ & $\begin{array}{l}60(77.9) \\
80(82.5) \\
13(59.1)\end{array}$ & $\begin{array}{l}1(1.3) \\
2(2.1) \\
0(0.0)\end{array}$ & $\begin{array}{c}16(20.8) \\
15(15.5) \\
9(40.9)\end{array}$ & $\begin{array}{c}10(90.9) \\
73(89.0) \\
6(85.7)\end{array}$ & $\begin{array}{c}1(9.1) \\
9(11.0) \\
1(14.3)\end{array}$ \\
\hline
\end{tabular}

Table 1: Infant Feeding Characteristics of Vegetarians

Previous breastfeeding practice was very high and varied among the women with respect to the type of vegetarian they are, education, race/ethnicity, marital status and region of residence in the United States as shown in Table 1, although the differences were not statistically significant. Rate of breastfeeding intention ranged from $78 \%$ to $82 \%$ for participants with bachelor's degree or higher compared to those with less than bachelor's degree. Similarly, the rate of previous breastfeeding practice ranged from $85 \%$ to $100 \%$ for participants with bachelor's degree or higher (Table 1). For the vegans who have had previous infant feeding experience, 9.1\% EBF for 3 months, $61.4 \%$ EBF for 6 months, while 13.6\% exclusively formula fed their child. Almost $65 \%$ of the lacto-ovo vegetarians reported to have EBF a previous child for 6 months. Flexitarians had the lowest rate of EBF a previous child for 6 months, while majority (55\%) of them MF their child. Although, rate of previous breastfeeding was high across the regions, Northeast, Southwest and Southeast recorded rates above $90 \%$ (Table 1).

There was no significant associations between vegans compared to the other vegetarian types for intention to breastfeed $(\mathrm{OR}=$ 0.62; 95\% CI: 0.29, 0.1.34; $\mathrm{p}=0.22)$ and previous breastfeeding practice $(\mathrm{OR}=1.64 ; 95 \% \mathrm{CI}: 0.47,5.78 ; \mathrm{p}=0.44)$.

\section{Discussion}

This pilot study adds to the evidence of infant feeding intentions and practices among different subgroups of the population, and the first among vegetarians, to the best of our knowledge. Our findings suggest that breastfeeding intentions and actual practice is overall relatively high among vegetarians who participated in this study. 
Findings from this study show that the majority of vegetarians who responded to this survey intend to breastfeed, and have a previous breastfeeding experience; however, their intention to exclusively breastfeed for 6 months is higher than previous exclusive breastfeeding practice although higher than the current rates among the general population in the United States [15]. This is an interesting finding that requires further exploration with a larger and more representative sample to understand why vegetarians have higher breastfeeding intentions and actual practice compared to the general US population.

In comparison to breastfeeding trends in the US, our data show that vegetarians may have a higher rate of breastfeeding. National data show that $79.2 \%$ of US women initiate breastfeeding [15,16], compared to $89 \%$ of vegetarians in this study. In fact, the rate of any breastfeeding was over $80 \%$ irrespective of the type of vegetarian and exceeds the breastfeeding initiation target of the Healthy People 2020 objective for breastfeeding [5]. National data show that 18.8\% of women exclusively breastfed for 6 months [15]; however, in this study, the rate was between $35 \%$ and $75 \%$ depending on the type of vegetarianism (data not shown). These findings could be a result of the motivation to become a vegetarian and majority of the participants being Caucasian. As the literature suggest, majority of individuals who choose vegetarianism do so based on the health benefits of the vegetarian diet [17]. This same health benefit may be the reason for the high breastfeeding intentions and practice observed in the current study among vegetarians, although not directly examined. With the majority of our participants reporting ethical reasons for their vegetarianism, we speculate that the high rates of breastfeeding intention and previous breastfeeding practice in the current study may be due to the likely opposition to infant formula because they are generally made from animal milk.

A surprising finding from this study is the higher breastfeeding rate among vegetarians in the southeast region of the United States. This observation is contrary to the breastfeeding trends of the general population in the southeastern United States [15]. The recent Breastfeeding Report Card by the CDC shows that the southern States have the lowest while the western States have the highest breastfeeding rates and duration [15]. Other studies that used nationally representative samples also observed higher breastfeeding rates in the west and northeast regions of the United States while the southern region recorded lowest rates of breastfeeding $[18,19]$. This finding among vegetarians in the southeast region of the United States may need further exploration to ascertain how it could benefit the general population.

Educated individuals are more likely to participate in healthy life-choices, such as breastfeeding [20]. A notable trend that has been observed is that the more education attained, the more likely women are to breastfeed $[2,16,19]$. This is confirmed in the current study with breastfeeding intentions and previous breastfeeding experience being higher among our highly educated participants.

There were several limitations to this study. Targeting a small subset of the general population spread across the country, such as vegetarians, proved to be challenging, and resulted in a smaller than anticipated sample size, which may explain our inability to observe statistically significant associations. It is important to note that the participants were largely homogenous: most had a higher level of education, were Caucasian, employed, married, and did not smoke. These characteristics could have a two-fold effect on the study findings. First, these characteristics have been found in national level studies with a stronger likelihood of breastfeeding $[16,21]$. This may have had a stronger influence on breastfeeding intentions and practice than the dietary practice of the participants as observed in the current study. Second, the homogenous nature of our participants does not make it representative of the general vegetarian sub-population in the United States, therefore limiting the generalizability of our findings. Selection bias may be a problem because the vegans $(n=102)$ and lacto-ovo vegetarian $(n=81)$ groups outnumbered the participants in the other vegetarian groups as well as only 2 blacks responding to the survey. These biases and limitations could have affected the internal validity of our findings as well as the generalizability of the results to the vegetarian sub-population in the United States.

Despite these limitations, this study did have its strengths. To the best of our knowledge, this cross-sectional online survey is the first to collect information about vegetarians and their breastfeeding intentions and practices in the United States. Most importantly, we received responses from all regions (38 States and the District of Columbia) of the continental United States, although the numbers were lower than we anticipated.

\section{Conclusion}

In summary, this study shows that the majority of vegetarians who participated intends to breastfeed and have previous breastfeeding experience. There were no statistical differences in the actual practice of breastfeeding between the vegetarian groups. This study also observed that the Caucasian participants, and those who were more educated, married, and resided in the southern region of the continental United States, had higher rates of breastfeeding intentions and previous breastfeeding experience, even though not statistically significant. Future research in this area with diverse and larger sample size is warranted to understand why vegetarians have high breastfeeding rates in order to determine the potential implications for public health and the Healthy People 2020 objectives for breastfeeding in the United States.

\section{Acknowledgement}

We thank all the listserv administrators who cooperated with us to forward the survey link to their membership. We also are very appreciative to all the participants of this study for taking the time to complete the survey. 


\section{References}

1. Johnston M, Landers S, Noble L, Szucs K, Viehmann L (2012) Breastfeeding and the use of human milk. Pediatrics 129: e827-41.

2. James DC, Lessen R, American Dietetic Association (2009) Position of the American Dietetic Association: Promoting and supporting breastfeeding. J Am Diet Assoc 109: 1926-42.

3. World Health Organization (1989) Protecting, Promoting, and Supporting Breastfeeding: The Special Role of Maternity Services, Geneva, Switzerland.

4. World Health Organization (2001) The optimal duration of exclusive breastfeeding: report of an expert consultation, Geneva, Switzerland.

5. U.S. Department of Health and Human Services, Healthy People 2020 Objectives: Maternal, Infant, and Child Health.

6. Ogbuanu CA, Probst J, Laditka SB, Liu JH, Baek JD, et al. (2009) Reasons why women do not initiate breastfeeding: A Southeastern State Study. Womens Health Issues 19: 268-78.

7. Society V (2014) The Vegetarian Society.

8. Ruby MB (2012) Vegetarianism. A blossoming field of study. Appetite 58: 141-50.

9. Newby PK, Tucker KL, Wolk A (2005) Risk of overweight and obesity among semivegetarian, lactovegetarian, and vegan women. Am J Clin Nutr 81: 1267-74.

10. Dyett PA, Sabate J, Haddad E, Rajaram S, Shavlik D (2013) Vegan lifestyle behaviors: an exploration of congruence with health-related beliefs and assessed health indices. Appetite 67: 119-24.

11. Euromonitor International (2011) The War on Meat: How Low-Meat and No-Meat Diets Impacting Consumer Markets. Euromonitor.

12. Newport F (2012) In U.S., 5\% Consider themselves Vegetarians, USA.

13. Vegetarian Times (2015) Vegetarianism in America.

14. Jensen T (2013) Food issues polarizing America public policy polling. Public Policy Polling, USA.

15. Centers for Disease Control and Prevention (2014) Breastfeeding Report Card, USA.

16. Centers for Disease Control and Prevention (2014) Breastfeeding among US children born 2001-2011, USA.

17. Pilis W, Stec K, Zych M, Pilis A (2014) Health benefits and risk associated with adopting a vegetarian diet. Rocz Panstw Zakl Hig 65: 9-14.

18. Kogan MD, Singh GK, Dee DL, Belanoff C, Grummer-Strawn LM (2008) Multivariate analysis of state variation in breastfeeding rates in the United States. Am J Public Health 98: 1872-80.

19. Li R, Ogden C, Ballew C, Gillespie C, Grummer-Strawn L (2002) Prevalence of Exclusive Breastfeeding Among US Infants: The Third National Health and Nutrition Examination Survey (Phase II, 1991-1994). Am J Public Health 92: 1107-10.

20. Taveras EM, Capra AM, Braveman PA, Jensvold NG, Escobar GJ, et al. (2003) Clinician support and psychosocial risk factors associated with breastfeeding discontinuation. Pediatrics 112: 108-15.

21. McDowell MM, Wang CY, Kennedy-Stephenson J (2008) Breastfeeding in the United States: findings from the national health and nutrition examination surveys, 1999-2006. NCHS Data Brief 1-8.

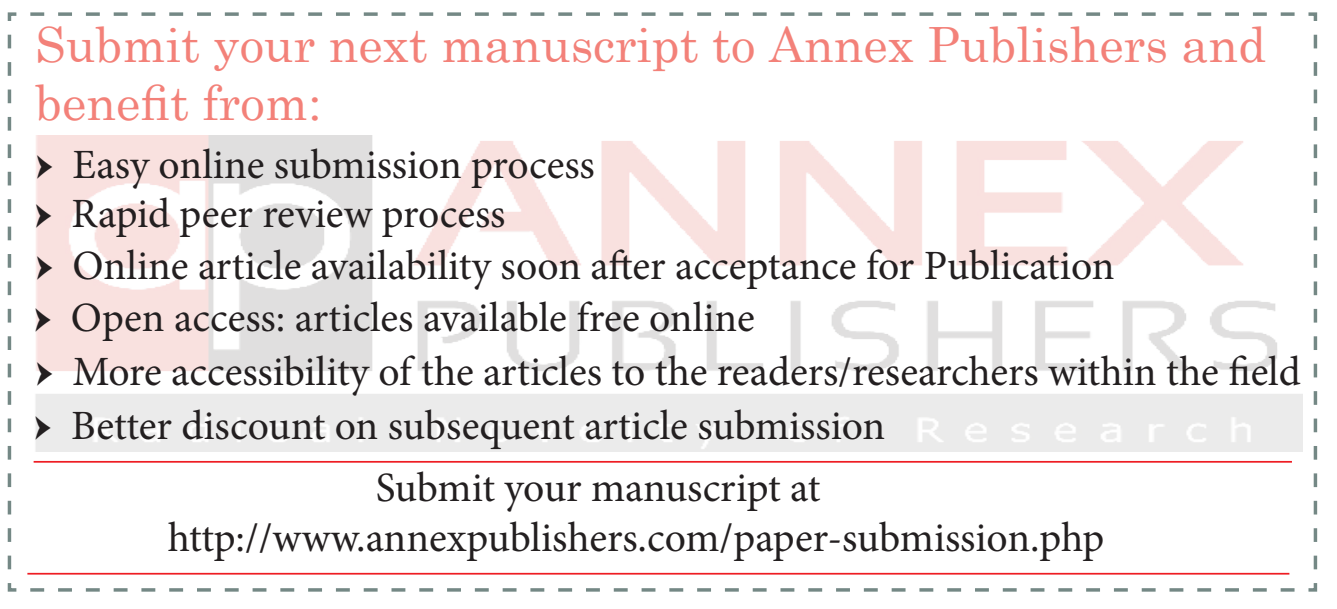

